\title{
Cash - Neue Dimension der Steuerung?
}

\section{Liebe Leserinnen und Leser,}

wie misst und steuert man den Erfolg eines Unternehmens? Generationen von BWL-Studentinnen und -Studenten haben im Wesentlichen das Gleiche gelernt: Sowohl im externen wie auch im internen Rechnungswesen geht es um Periodenerfolge. Hiermit sind diverse Probleme verbunden, wie schon Wilhelm Rieger in seinem berühmten Zitat ausgeführt hat: Jede Zwischenbilanz gerät „mit dem lebendigen Betrieb und seinen Rhythmen [in Konflikt]. Sie zertrennt rechnungsmäßig mit der Rücksichtslosigkeit einer Guillotine feinste betriebliche Zusammenhänge - und dies nennt man Abschluss" (Rieger, W.: Einführung in die Privatwirtschaftslehre, 1. Aufl., Erlangen 1929, S. 210). Dennoch hat sich der Periodenerfolg auf ganzer Linie durchgesetzt. Wie man aus erfolgswirksamen Zahlungsgrößen durch Periodisierung Aufwendungen und Erträge bzw. Kosten und Erlöse macht, füllt ganze Bibliotheken.

Wenn Studierende mit zahlungsorientierten Erfolgsmaßen in Berührung kommen, so ist das zumeist im Bereich von Investitionsprojekten der Fall. Diese sind nicht an die Rasterung der Abrechnungsperioden gebunden. Bei ihnen besteht nicht die Notwendigkeit zur unterjährigen Periodenaufteilung. Letztere wird erst dann wirksam, wenn die Investition in das Regelgeschäft integriert ist. Dann sehen wir sie unter anderem in Form von Abschreibungen wieder.

Neben der periodischen Erfolgsrechnung steht losgelöst eine Zahlungs- bzw. Liquiditätsrechnung. Diese hat in der Vergangenheit für Controller keine nennenswerte Rolle gespielt. Für Finanzierungsfragen waren andere zuständig. Auch bei einer Investition ging es Controllern nur um den Kapitalwert. Ob die Investition bei einem positiven Kapitalwert auch finanzierbar war, interessierte sie nicht („das Geld kam aus der Steckdose").

Seit einiger Zeit ist diese alte Position ins Wanken geraten. Vielerlei Einflüsse sind dafür maßgeblich. Einen Frontalangriff auf das Periodenerfolgskonzept gab es in den 1980er Jahren aus Richtung des Shareholder Value Managements. Anfänglich wurde vorgeschlagen, den Wert des Unternehmens mittels der Discounted-Cashflow-Methode (DCFMethode) zu ermitteln. Der Erfolg einer Periode war dann die Entwicklung dieses DCFs innerhalb eines Jahreszeit-

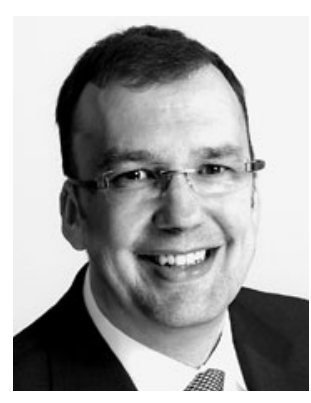

Utz Schäffer

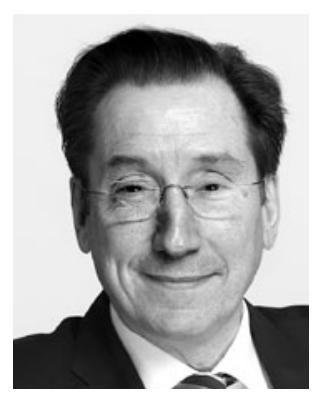

Jürgen Weber raums. Allerdings zeigte sich schnell, dass ein solches Vorgehen in seiner Operationalisierung zu große praktische Probleme mit sich brachte, sodass eher pragmatische und stärker mit der traditionellen Denkweise kompatible Alternativen wie z. B. der EVA entwickelt wurden. Obwohl sie wieder der klassischen Periodenlogik folgen, betonen sie die Bedeutung des im Unternehmen gebundenen risikotragenden Kapitals deutlich stärker. In der Folge wurde die Kapitalbindung in den Unternehmen ein wichtiges Steuerungsobjekt für die Controller. Für viele von ihnen sind etwa Working-Capital-Projekte ein etablierter Bestandteil ihres laufenden Aufgabenportfolios.

Ein weiterer Treiber steigender Cash-Orientierung ist die zunehmende Kapitalknappheit auf der Fremdkapitalseite. Geld ist nicht mehr so leicht zu beschaffen, der dafür zu zahlende Zins ist stark abhängig vom Risiko des Unternehmens und seiner Kapitalstruktur. Entsprechende neue Zielgrößen (Einhaltung von „covernants") sind entstanden. Controller können sich heute nicht mehr nur auf die periodischen Erfolge beschränken, sondern müssen stets auch die Kapitalstruktur mitbedenken - auch bei einzelnen Investitionen. Das Geld kommt nicht mehr automatisch aus der Steckdose.

Ein dritter, wesentlicher Einfluss stammt von einer Branche, der ein eher schlechtes Image anhaftet. Oft wurden Private-Equity-Gesellschaften verteufelt - man erinnere sich an das bekannte Zitat der Heuschrecken von Franz Müntefering. Schaut man genauer hin, hat sich das Wirken von Private Equity oftmals allerdings als durchaus segensreich heraus- 
gestellt. Private-Equity-Gesellschaften betrachten ein Unternehmen als eine Investition, die sich rechnen muss und die keinen Anspruch darauf hat, unsterblich zu sein. Das Lebenszykluskonzept ist in der BWL weit verbreitet, ebenso wie das Ziel, die Überlebensfähigkeit von Unternehmen zu sichern. Diese Analogie hinkt allerdings ein wenig. Unternehmen müssen Ressourcen wirtschaftlich verwenden. Wenn sie das nicht mehr oder andere es besser können, hilft es der Gesellschaft, wenn sie vom Markt verschwinden. Private Equity hat häufig dazu geführt, dass gerade ein solches Verschwinden vermieden werden konnte, da die Unternehmen wieder wettbewerbsfähig wurden. Für Private-Equity-Investoren sind Zahlungen die wichtigere Größe als der Periodenerfolg. Controller können sich hier also durchaus ein Beispiel nehmen. Diese Perspektive liefert erfahrungsgemäß erfrischend neue Einsichten, wenn sie konsequent eingenommen wird.
Wir wollen unterm Strich kein Plädoyer dafür halten, dass die Zahlungsperspektive in der Erfolgsrechnung das traditionelle Periodenerfolgskonzept ersetzen sollte. Wir wollen Sie aber ermuntern, die Cash-Perspektive als ergänzende Sichtweise auf den Unternehmenserfolg zu betrachten - nicht nur fallweise, sondern regelmäßig. Dies bedeutet für die meisten Controller ein erhebliches Dazulernen. Es lohnt sich, weil es in den meisten Fällen zu neuen Erkenntnissen führt, die das Unternehmen dringend braucht. Kapital wird knapp bleiben und die wirtschaftliche Dynamik noch weiter ansteigen - zwei Gründe für ein „Sowohl-als-auch“!

Viel Spaß bei der Lektüre dieses Heftes wünschen Ihnen
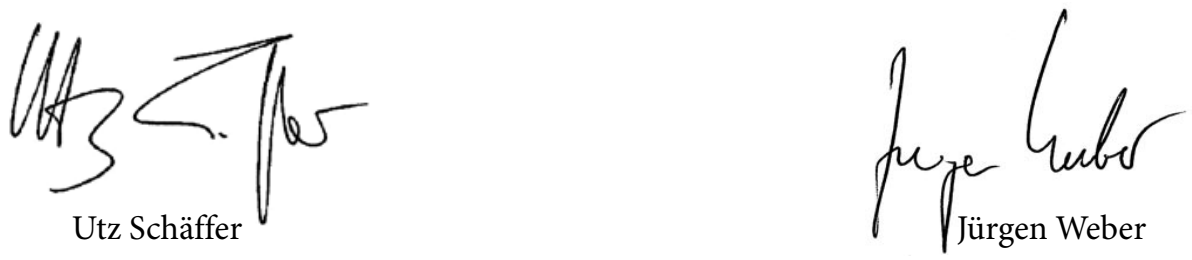


\section{Springer Gabler}

\section{Thomas Kapp}

\section{Kartellrecht in der Unternehmens- praxis}

Was Unternehmer und Manager wissen müssen

2. Auflage

\section{Das erste Kartellrechtsbuch für Praktiker.}

\section{Thomas Kapp}

Kartellrecht in der Unternehmenspraxis Was Unternehmer und Manager wissen müssen

2013. XII, 330 S. 4 Abb. Brosch.

$€$ (D) $59,99|€(A) 61,67|{ }^{*} \mathrm{sFr} 75,00$

ISBN 978-3-8349-3028-6

\section{- Die neue 8. GWB-Novelle bereits eingearbeitet}

Kartellrechtliche Fragen betreffen auch Inhaber, Vorstände und Geschäftsführer mittelständischer Unternehmen, nicht nur Großunternehmen und Konzerne. Hier setzt der ausgewiesene Kartellanwalt Thomas Kapp an. Das Buch ist ein Handbuch für Praktiker. Unter Verzicht auf juristische Detaildiskussion gibt er Unternehmern, Vorständen, Geschäftsführern, Aufsichtsräten und Rechtsberatern einen praktischen Leitfaden zur schnellen Erfassung kartellrechtlicher Fragestellungen (Kartellverbot, Bußgeldverfahren, Missbrauchskontrolle, Fusionskontrolle, Privater Rechtsschutz) an die Hand und bietet Lösungswege an. Praxisfälle, Checklisten und Illustrationen erläutern und verdeutlichen die behandelten Sachverhalte. Auf die Trennung zwischen europäischem und deutschem Recht wird zu Gunsten der besseren Lesbarkeit weitgehend verzichtet. Die Neuregelungen der 8. GWB-Novelle werden bereits berücksichtigt. 